school flunking ( $\mathrm{PR}=1.23$, 95\% CI 1.01 to 1.51) or aggressive behaviour ( $\mathrm{PR}=1.62,95 \% \mathrm{CI} 1.36$ to 1.94$)$. The association between self-reported discrimination and nutrition status (using BMI-for-age $z$-score) varied according to sex ( $p$ for interaction $=0.009$ ). Thin boys were more likely to report discrimination ( $\mathrm{PR}=1.94,95 \% \mathrm{CI} 1.05$ to 3.56), while overweight and obese ones showed lower prevalence $(\mathrm{PR}=0.65$ and 0.67 , respectively). Higher prevalence of discrimination was observed in obese girls ( $P R=1.54,95 \%$ CI 1.12 to 2.10 ), and this effect was stronger among wealthiest than in the poorest females ( $P R=2.14$ and 1.48, respectively; $p$ for interaction 0.083 ).

Conclusions Self-reported discrimination was prevalent, and unevenly distributed among the population. Interventions to reduce discriminatory experiences should be implemented in early stages of the life cycle.

\section{P2-406 INFLUENCE OF SKIN COLOUR AND LIFE-COURSE SOCIOECONOMIC POSITION ON ABDOMINAL OBESITY AMONG ADOLESCENTS IN A BRAZILIAN BIRTH COHORT}

doi:10.1136/jech.2011.142976l.36

${ }^{1,2}$ D A G Chica, ${ }^{* 2}$ A M B Menezes, ${ }^{3} \mathrm{~A}$ Nazmi, ${ }^{2} \mathrm{C}$ G Victora. ${ }^{1}$ Universidade Federal de Santa Catarina, Florianópolis, Santa Catarina, Brazil, ${ }^{2}$ Universidade Federal de Pelotas, Pelotas, Rio Grande do Sul, Brazil; ${ }^{3}$ Department of Food and Nutrition, California Polytechnic State University, San Luis Obispo, California, USA

Objective To evaluate the effects of skin colour and life-course socioeconomic indicators on waist circumference (WC) and waistto-height ratio (WHtR) in adolescence in a population-based birth cohort study.

Methodology All the 5249 individuals born in Pelotas (southern Brazil) in 1993 were repeatedly visited from birth to age $15 \mathrm{y}$. In 2008 the whole cohort was traced. The analysis was restricted to individuals located and measured at age 15 y (2004 males, 2094 females).

Results WC was higher in men that in women $(72.4$ and $68.9 \mathrm{~cm}$, respectively, $p<0.001)$, but WHtR showed no difference $(0.43$ in both cases, $p=0.9)$. In men, family income at birth and at age $15 \mathrm{y}$ were positively associated with WC, but only the former was associated with WHR. After adjustment for current family income and maternal education, men born to better-off families remained with larger WC in adolescence, but the association with WHtR was missed. Skin colour was not associated with any outcome. In women, neither skin colour nor family income (at birth or at age $15 \mathrm{y}$ ) were associated with WC or WHtR. All the associations in men remained even after adjustment for adolescent's behavioural variables (physical activity, fat intake, smoke and alcohol intake).

Conclusions In men, early and current socioeconomic position are directly associated with abdominal obesity. The effects of early socioeconomic conditions on WC persist even after adjustment for maternal education, adult wealth and current behavioural variables, highlighting the importance of interventions during the first years of life.

\section{P2-407 PERCEIVED SOCIAL SUPPORT AND LEISURE-TIME PHYSICAL ACTIVITY LEVELS IN ADULTS LIVING IN A LARGE URBAN CENTER: SAÚDE EM BEAGÁ STUDY - BELO HORIZONTE CITY, BRAZIL}

doi:10.1136/jech.2011.142976l.37

\footnotetext{
1,2J Goston, ${ }^{* 2} \mathrm{~A}$ Andrade, ${ }^{1,2} \mathrm{~F}$ Proietti, ${ }^{1,2} \mathrm{~W}$ Caiaffa. ${ }^{1}$ Federal University of Minas Gerais, School of Medicine, Public Health, Belo Horizonte, Minas Gerais, Brazil; ${ }^{2}$ Observatory for Urban Health, Epidemiology Research Group, Public Health, Belo Horizonte, Minas Gerais, Brazil

Introduction Leisure Physical Activity (LPA) is an important health determinant. Alarming rates of sedentarism are observed in world's population.
}

Objective To investigate the influence of Social Support for Physical Activity (PASS) and individual factors on the LPA levels in adults of a large urban center.

Methods Household survey was carried out with 4048 adults. Demographic, social determinants and health information were collected. The LPA levels were categorised into inactive, insufficiently active and active, according to the International Physical Activity Questionnaire. The PASS scale was constructed using three indicators: PASS neighbourhood, encouragement and commitments from friends and family, with responses ranging from "no" to "high support". Associations between LPA levels and PASS were investigated using multivariate ordinal logistic model.

Results Our study consisted of 3453 adults aged $18-69$ yo (1595 men; 1858 women); $59.9 \%$ ( $n=2171)$ were classified into inactive, $23.3 \%(n=727)$ insufficiently active, and $16.8 \%(n=555)$ sufficiently active. Participants were more likely to be male $(\mathrm{OR}=1.53)$, no partners $(\mathrm{OR}=1.30)$, education level higher than nine years $(\mathrm{OR}=1.97)$, and highest family income ( $\geq 5$ minimum wage) $(\mathrm{OR}=1.76)$, were more likely to be in a better level of $L P A$. Moreover, those participants no PASS were more likely to be in a worse $L P A$ levels than those with highest PASS (low: $O R=1.80$; medium: $O R=2.51$; high: $O R=2.73$ )

Conclusion Participants with highest PASS were more likely to achieve the current recommendation for LPA ( $\geq 150 \mathrm{~min} /$ week). Social Support may be an important aspect for Physical Activity interventions and some encouragement from family and friends may have a positive impact in the behaviours changes.

\section{P2-408 PREVALENCE OF AND ATTITUDES TOWARDS SMOKING AMONG MIDWIFES IN NORTHWEST RUSSIA}

doi:10.1136/jech.2011.142976l.38

${ }^{1} 0$ Kharkova, ${ }^{2}$ A Soloviev, ${ }^{3,4}$ A Grjibovski. ${ }^{*}$ International School of Public Health, Northern State Medical University, Arkhangelsk, Russia; ${ }^{2}$ Northern State Medical University, Arkhangelsk, Russia; ${ }^{3}$ Norwegian Institute of Public Health, Oslo, Norway; ${ }^{4}$ Institute of Community Medicine, University of Tromsø, Tromsø, Norway

Introduction Smoking is the leading cause of premature mortality and Russia is among the countries with the highest prevalence of smoking. Pregnancy is considered as a good time-point for smoking cessation and midwifes can play an important role in this process. However, it is generally unknown how prevalent is smoking among midwifes in Russia. The aim is to assess the prevalence of and attitudes towards smoking among midwifes in Northwest Russia. Methods A questionnaire on smoking and attitudes was sent to all 83 midwifes in the city of Arkhangelsk and $72 \%$ of them responded. All data were analysed using $\chi^{2}$ tests.

Results The prevalence of smoking among midwifes was $43.5 \%$. Eighty-five percent of midwifes considered that it is necessary to implement antismoking program in antenatal clinics. Most midwifes $(72.6 \%)$ did not agree that nurses should be involved in smoking prevention program for pregnant women. Only $17 \%$ of smokers counselled women on smoking cessation while among nonsmokers this proportion was $52.0 \%(p=0.024)$. Surprisingly, $67.0 \%$ of midwifes stated that it might be harmful to immediately quit smoking during pregnancy. Midwifes reflected lack of knowledge to counsel pregnant women about tobacco. Many could not list main negative effects of smoking. Only $35.1 \%$ of midwifes mentioned that smoking during pregnancy can cause fetoplacental insufficiency, $24.6 \%$ named low birth weight and $5.0 \%$ mentioned miscarriage, intrauterine infections and lung diseases in infants.

Conclusions A high proportion of midwifes are smokers themselves and many are poorly informed about effects of smoking. 